\title{
Optimal Portfolio Problem for Stochastic-Volatility, Jump-Diffusion Models with Jump-Bankruptcy Condition: Practical Theory
}

\author{
BFS 2008 Full Paper \\ Floyd B. Hanson \\ Department of Math., Stat., and Computer Science, M/C 249 \\ University of Illinois at Chicago \\ hansonemath.uic.edu
}

January 10, 2008

\begin{abstract}
This paper treats the risk-averse optimal portfolio problem with consumption in continuous time with a stochastic-volatility, jump-diffusion (SVJD) model of the underlying risky asset and the volatility. The new developments are the use of the SVJD model with double-uniform jumpamplitude distributions and time-varying market parameters for the optimal portfolio problem. Although unlimited borrowing and short-selling play an important role in pure diffusion models, it is shown that borrowing and short selling are constrained for jump-diffusions. Finite range jump-amplitude models can allow constraints to be very large in contrast to infinite range models which severely restrict the optimal instantaneous stock-fraction to $[0,1]$. The reasonable constraints in the optimal stock-fraction due to jumps in the wealth argument for stochastic dynamic programming jump integrals remove a singularity in the stock-fraction due to vanishing volatility. Main modifications for the usual constant relative risk aversion (CRRA) power utility model are for handling the partial integro-differential equation (PIDE) resulting from the additional variance independent variable, instead of the ordinary integro-differential equation (OIDE) found for the pure jump-diffusion model of the wealth process. In addition to natural constraints due to jumps when enforcing the positivity of wealth condition, other constraints are considered for all practical purposes under finite market conditions. Also, a computationally practical solution of Heston's (1993) square-root-diffusion model for the underlying asset variance is derived. This shows that the nonnegativity of the variance is preserved through the proper singular limit of a simple perfect-square form. An exact, nonsingular solution is found for a special combination of the Heston stochastic volatility parameters.
\end{abstract}

Key words: optimal-portfolio problem, stochastic-volatility, jump-diffusion, finite markets, jump-bankruptcy condition, double-uniform jump-amplitudes, nonnegative-variance verification.

\section{Introduction}

The empirical distribution of daily log-returns for real financial investments differs in many ways from the ideal pure diffusion process with its log-normal distribution as assumed in the Black- 
Scholes-Merton option pricing model $[10,46]$. One of the most significant differences is that actual log-returns exhibit occasional large jumps in value, whereas the diffusion process in BlackScholes [10] is continuous. Statistical evidence of jumps in various financial markets is given by Ball and Torous [7], Jarrow and Rosenfeld [34], and Jorion [36]. Long before this statistical-jump evidence, Merton [47] (also [48, Chap. 9]) published a pioneering jump-diffusion model using lognormal jump-amplitudes. Other jump-diffusion models were proposed including Kou and Wang's log-double-exponential [39, 40] and Hanson and Westman's log-uniform [26, 28] jump-diffusion models or Zhu and Hanson's log-double-uniform model [58, 59]. However, it is difficult to separate the outlying jumps from the diffusion, although separating out the diffusion is a reasonable task as shown by Aït-Sahalia [1].

Another difference is that the empirical log-returns are usually negatively skewed, since the negative jumps or crashes are likely to be larger or more numerous than the positive jumps for many instruments over sufficiently long periods, whereas the normal distribution associated with the logarithm of the diffusion process is symmetric and hence has zero skew. A third difference is that the empirical distribution is usually leptokurtic, since the coefficient of kurtosis, i.e., the variancenormalized fourth central moment [16], is bounded below by the normal distribution kurtosis value of three. Qualitatively, this means that the tails are fatter than a normal with the same mean and standard deviation, compensated by a distribution that is also more slender about the mode (local maximum).

A fourth difference is that the market exhibits time-dependence in the distributions of log-returns, so that the associated parameters are time-dependent. In particular, another significant difference is the volatility, which is time-dependent and stochastic, i.e., we have stochastic volatility. Stochastic volatility in the market, mostly in options pricing, has been studied by Garman and Klass [19], Johnson and Shanno [35], Ball and Torous [6], Hull and White [32], Wiggins [54], Stein and Stein [53, see corrections in [5]], Ball and Roma [5], Scott [52], and Lord, KoekKoek and Dijk [41]. The mean-reverting, square-root-diffusion, stochastic-volatility model of Heston [21] is frequently used. Heston's model derives from the CIR model of Cox, Ingersoll and Ross [13] for interest rates. The CIR paper also cites the Feller [17] justification for proper (Feller) boundary conditions, process nonnegativity and the distribution for the general square-root diffusions. In a companion paper to the CIR model paper, Cox et al. [12] present the more general theory for asset processes. In their monograph, Fouque, Papanicolaou and Sircar [18] cover many issues involving various models with stochastic volatility. Andersen, Benzoni and Lund [2], as well as others, have statistically confirmed the importance of both stochastic volatility and jumps in equity returns. In their often cited paper on affine jump-diffusions, Duffie, Pan and Singleton [15] include a section on various stochasticvolatility, jump-diffusion models. Bates [9] studied stochastic-volatility, jump-diffusion models for exchange rates. Broadie and Kaya [11] devised an exact simulation method for stochastic-volatility, affine-jump-diffusion models for option pricing in the sense of an unbiased Monte Carlo estimator. Yan and Hanson [55, 56, 30] explored theoretical and computational issues for both European and American option pricing using stochastic-volatility, jump-diffusion models with log-uniform jumpamplitude distributions.

For the optimal portfolio with consumption problem, Merton [44, 45] (see also [48, Chapters 4-6]), in a prior pioneering paper, analyzed the optimal consumption and investment portfolio with geometric Brownian motion and examined an example of hyperbolic absolute risk-aversion (HARA) utility having explicit solutions. Generalizations to jump-diffusions consisting of Brownian motion and compound Poisson processes with general random finite amplitudes are briefly discussed. Earlier in [43] ([48, Chapter 4]), Merton also examined constant relative risk-aversion problems.

In the 1971 Merton paper [44, 45] there are a number of errors, in particular in boundary conditions for bankruptcy (negative wealth) and vanishing consumption. Some of these problems are 
directly due to using a general form of the HARA utility model. These errors are very thoroughly discussed in a seminal collection assembled by Sethi [50] from his papers and those with his coauthors. Sethi in his introduction [50, Chapter 1] thoroughly summarizes these errors and subsequent generalizations. In particular, basic papers of concern here are the KLSS paper with Karatzas, Lehoczhy, Sethi and Shreve [37] (reprint [50, Chapter 2]) for exact solutions in the infinite horizon case and with Taksar [51] (reprint [50, Chapter 2]) pinpointing the errors in Merton's [44, with [45] erratum] work.

Hanson and Westman [23, 29] reformulated an important external events model of Rishel [49] solely in terms of stochastic differential equations and applied it to the computation of the optimal portfolio and consumption policies problem for a portfolio of stocks and a bond. The stock prices depend on both scheduled and unscheduled jump external events. The complex computations were illustrated with a simple log-bi-discrete jump-amplitude model, either negative or positive jumps, such that both stochastic and quasi-deterministic jump magnitudes were estimated. In [24], they constructed a jump-diffusion model with marked Poisson jumps that had a log-normally distributed jump-amplitude and rigorously derived the density function for the diffusion and log-normal-jump stock price log-return model. In [25], this financial model is applied to the optimal portfolio and consumption problem for a portfolio of stocks and bonds governed by a jump-diffusion process with log-normal jump amplitudes and emphasizing computational results. In two companion papers, Hanson and Westman [26, 27] introduce the log-uniform jump-amplitude jump-diffusion model, estimate the parameter of the jump-diffusion density with weighted least squares using the S\&P500 data and apply it to portfolio and consumption optimization. In [28], they study the time-dependence of the jump-diffusion parameter on the portfolio optimization problem for the log-uniform jump-model. The appeal of the log-uniform jump model is that it is consistent with the stock exchange introduction of circuit breakers [3] in 1988 to limit extreme changes, such as occurred in the crash of 1987, in stages. On the contrary, the normal [47, 2, 24] and double-exponential jump [39, 40] models have an infinite domain, which is not a problem for the diffusion part of the jump-diffusion distribution since the contribution in the dynamic programming formulation is local appearing only in partial derivatives. However, the influence of the jump part in dynamic programming is global through integrals with integrands that have shifted arguments. This has important consequences for the choice of jump distribution since the portfolio wealth restrictions will depend on the range of support of the jump density.

However, there has been much less effort on the optimal portfolio with consumption problem when stochastic volatility is included, and what is available tends to be very theoretical in nature. Cox, Ingersoll and Ross [12] consider the very general optimal portfolio with consumption problem for a very general state vector that could include stochastic volatility and a von NeumannMorganstern utility, and in the CIR model paper [13] considered the special case of the logarithmic utility. Wiggins [54] considers the optimal portfolio problem for the log-utility investor with stochastic volatility and using equilibrium arguments for hedging. Zariphopoulou [57] analyzes the optimal portfolio problem with CRRA utility, a stochastic factor, i.e., stochastic volatility, and unhedgeable risk.

In this paper, the log-double-uniform jump-amplitude, jump-diffusion asset model with a Heston model stochastic volatility is applied to the portfolio and consumption optimization problem. In Section 2, the stochastic-volatility, jump-diffusion model is formulated as the underlying twodimension process for the optimal portfolio and consumption problem. In Section 3, the portfolio optimization with consumption problem is formulated by stochastic dynamic programming and jump-no-bankruptcy conditions are derived. In Section 4, the canonical solutions for CRRA power and logarithmic utilities are derived using a implicit type of Bernoulli transformation. In Section 5, the preservation of positivity of the optimal wealth from positive initial wealth is formally justified. 
In Section 6, the nonnegativity of the variance is verified using a proper singular limit of a perfectsquare form and an exact, nonsingular solution is given for special values of the Heston model [21] stochastic-volatility parameters. In Section 7, conclusions are drawn.

\section{Optimal portfolio problem and underlying SVJD model}

Let $S(t)$ be the price of a single underlying financial asset, such as a stock or mutual fund, governed by a Markov, geometric jump-diffusion stochastic differential equation with time-dependent coefficients,

$$
d S(t)=S(t)\left(\mu_{s}(t) d t+\sqrt{V(t)} d G_{s}(t)+\nu_{s}(V(t), t, Q) d P_{s}(t ; Q)\right),
$$

with $S(0)=S_{0}>0$, where $\mu_{s}(t)$ is the mean appreciation return rate at time $t, V(t)=\sigma_{s}^{2}(t)$ is the diffusive variance, $d G_{s}(t)$ is a continuous Gaussian process with zero mean and $d t$ variance (the usual symbol $W$ is used here for wealth and $B$ is used for the bond price), $d P_{s}(t ; Q)$ is a discontinuous, standard Poisson process with jump rate $\lambda_{s}(t)$, with common mean-variance of $\lambda_{s}(t) d t$, and associated jump-amplitude $\nu_{s}(v, t, q)$ with IID log-return mark $Q$ jump-mean $\mu_{j}(t)$ and jumpvariance $\sigma_{j}^{2}(t)$. The stochastic processes $G_{s}(t)$ and $P_{s}(t)$ are assumed to be Markov and pairwise independent. The jump-amplitude $\nu_{s}(V(t), t, Q)$, given that a Poisson jump in time occurs, is also independently distributed, at pre-jump time $T_{k}^{-}$and mark $Q_{k}$. In Eq. (2.1), the following short-hand notation is used,

$$
\nu_{s}(V(t), t, Q) d P_{s}(t ; Q) \equiv \sum_{k=P_{s}(t ; Q)+1}^{\left(P_{s}+d P_{s}\right)(t ; Q)} \nu_{s}\left(V\left(T_{k}^{-}\right), T_{k}^{-}, Q_{k}\right)
$$

provided $d P_{s}(t ; Q) \geq 1$, else the sum is defined as zero by convention, where $T_{k}^{-}$is the $k$ th pre-jump time. The $Q_{k}$ are IID random variables with jump-amplitude mark density $\phi_{Q}(q ; t)$ on the mark-space $\mathcal{Q}$.

There are many jump-amplitude distributions for the log-return that are used to define $\phi_{Q}(q ; t)$. Among them are the log-normal jump-amplitude distribution used by Merton [47] in his pioneering jump-diffusion finance paper (see also Hanson and Westman [25]), the log-double-exponential distribution used by Kou and coauthor $[39,40]$, and the log-uniform and log-double-uniform distributions used by Hanson and coauthors [26, 27, 58, 59, 56]. Since it is difficult to determine what the market jump-amplitude distribution is, the double-uniform distribution is the simplest distribution that clearly satisfies the critical fat-tail property and allows separation of crash and rally behaviors by the double composite property. So, let the log-double-uniform density be

$$
\phi_{Q}(q ; v, t) \equiv\left\{\begin{array}{ll}
0, & -\infty<q<a(v, t) \\
p_{1}(v, t) /|a|(v, t), & a(v, t) \leq q<0 \\
p_{2}(v, t) / b(v, t), & 0 \leq q \leq b(v, t) \\
0, & b(v, t)<q<+\infty
\end{array}\right\}
$$

where $a(v, t)<0<b(v, t), p_{1}(v, t) \geq 0$ is the probability of a negative jump and $p_{2}(v, t) \geq 0$ is the probability of a non-negative jump such that $p_{1}(v, t)+p_{2}(v, t)=1$. Otherwise, a well-defined form of the log-double-uniform distribution is

$$
\begin{aligned}
\Phi_{Q}(q ; v, t)= & p_{1}(v, t) \frac{q-a(v, t)}{|a|(v, t)} \mathcal{I}_{\{a(v, t) \leq q<0\}}+\left(p_{1}(v, t)+p_{2}(v, t) \frac{q}{b(v, t)}\right) \mathcal{I}_{\{0 \leq q<b(v, t)\}} \\
& +\mathcal{I}_{\{b(v, t) \leq q<\infty\}},
\end{aligned}
$$


where $\mathcal{I}_{\mathcal{S}}$ is the indicator function for set $\mathcal{S}$. Since the double-uniform jump-amplitude distribution is used here and elsewhere for the stock log-return variable and it is desirable to keep the mark variable as simple as possible, let the mark variable be the jump in the log-return [22], i.e.,

$$
Q \equiv \operatorname{Jump}[\ln (S)](t)=\ln \left(\left(1+\nu_{s}(V(t), t, Q)\right) S(t)\right)-\ln (S(t))=\ln \left(1+\nu_{s}(V(t), t, Q)\right)
$$

or

$$
\nu_{s}(v, t, q) \equiv e^{q}-1
$$

leaving the $v$ and $t$ dependence in $a(v, t)$ and $b(v, t)$

The stock price SDE (2.1) is similar in prior work [24, 25], except that time-dependent coefficients introduce more realism here as used in [59].

The stochastic variance is modeled with the Cox-Ingersoll-Ross (CIR) $[12,13]$ and Heston [21] mean-reverting stochastic volatility, $\sigma_{s}(t)=\sqrt{V(t)}$, and square-root diffusion with parameters $\left(\kappa_{v}(t), \theta(t), \sigma_{v}(t)\right)$ :

$$
d V(t)=\kappa_{v}(t)\left(\theta_{v}(t)-V(t)\right) d t+\sigma_{v}(t) \sqrt{V(t)} d G_{v}(t)
$$

with $V(0)=V_{0}>0$, log-rate $\kappa_{v}(t)>0$, reversion-level $\theta_{v}(t)>0$ and volatility of volatility (variance) $\sigma_{v}(t)>0$, where $G_{s}(t)$ and $G_{v}(t)$ are standard Brownian motions for $S(t)$ and $V(t)$, respectively, with correlation $\operatorname{Corr}\left[d G_{s}(t), d G_{v}(t)\right]=\rho$. It will be assumed that the volatility is nonnegative, i.e., $V(t) \geq 0$, but this will be qualified later. Equations (2.1) and (2.4) comprise the underlying stochastic-volatility, jump-diffusion (SVJD) model. See also [9, 52, 18, 55, 56, 30] for other applications.

The riskless asset with a variable interest rate yields variable deterministic exponential growth,

$$
d B(t)=r(t) B(t) d t
$$

where $B(0)>0$ and $r(t)$ is the interest rate.

The portfolio consists of the stock $S(t)$ and the bond $B(t)$ with instantaneous portfolio-fractions $U_{s}(t)$ and $U_{b}(t)$, respectively, such that $U_{b}(t)=1-U_{s}(t)$. The wealth $W(t)$ satisfies the selffinancing condition, so that

$$
\begin{gathered}
d W(t)=W(t) \cdot\left(r(t) d t+U_{s}(t) \cdot\left(\left(\mu_{s}(t)-r(t)\right) d t+\sqrt{V(t)} d G_{s}(t)\right.\right. \\
\left.\left.+\nu_{s}(V(t), t, Q) d P_{s}(t ; Q)\right)\right)-C(t) d t
\end{gathered}
$$

where $W(0)=W_{0}>0, U_{b}(t)$ has been eliminated and $C(t)$ is the instantaneous consumption. The portfolio $\mathrm{dS}(\mathrm{t})$ system consists of the wealth equation in (2.6) plus an additional equation for the variance (volatility) equation in (2.4) beyond the usual portfolio problem [43, 44]. The system is subject to constraints that there be no bankruptcy, $W(t) \geq 0$, that consumption cannot exceed a certain fraction of wealth, i.e.,

$$
0 \leq C(t) \leq C_{0}^{(\max )}(V(t), t) \cdot W(t)
$$

with $0<C_{0}^{(\max )}(V(t), t)<1$, and that there be no negative variance, $V(t) \geq 0$. Later, we will find an additional constraint on the stock-fraction as a consequence of the effect of jumps on the bankruptcy condition [59]. Note that our definition of bankruptcy $W(t)<0$ corresponds to that of Merton [44] and differs from the Karatzas et al. [37] definition $W(t)=0$, since that just means no 
wealth while $W(t)<0$ means that the investor is in debt. The instantaneous stock-fraction $U_{s}(t)$ is assumed to be bounded above and below.

The optimization criterion or performance index is the optimal, conditionally expected, discounted utility of final wealth plus the cumulative, discounted utility of running consumption,

$$
J^{*}(w, v, t)=\max _{u, c}\left[\mathrm{E}\left[e^{-\bar{\beta}\left(t ; t_{f}\right)} \mathcal{U}_{w}\left(W\left(t_{f}\right)\right)+\int_{t}^{t_{f}} e^{-\bar{\beta}(t ; \tau)} \mathcal{U}_{c}(C(\tau)) d \tau \mid \mathcal{C}\right]\right],
$$

where $\mathcal{C}=\left\{W(t)=w, V(t)=v, C(t)=c, U_{s}(t)=u\right\}$ is the conditioning, $\bar{\beta}(t ; \tau)=\int_{t}^{\tau} \beta(y) d y$ is the cumulative discount, $\beta(t)$ is the instantaneous discount, $\mathcal{U}_{w}(w)$ is the utility of the final wealth $w$ and $\mathcal{U}_{c}(c)$ is the utility of the instantaneous consumption $c$. The consumption $c$ and the stock-fraction $u$ are obviously the two control variables of the optimal portfolio problem and are derived as the arguments of the maximization.

There are several side conditions deducible from the criterion (2.8). As the final time is approached, $t \rightarrow t_{f}^{-}$, the final condition is obtained,

$$
J^{*}\left(w, v, t_{f}^{-}\right)=\mathcal{U}_{w}(w),
$$

for any final wealth level $w>0$. As the wealth approached zero, $w \rightarrow 0^{+}$, so does the consumption, $c \rightarrow 0^{+}$, since it is constrained as a fraction of wealth and by definition zero wealth is an absorbing boundary with boundary condition, from the objective (2.8),

$$
J^{*}\left(0^{+}, v, t\right)=\mathcal{U}_{w}\left(0^{+}\right) e^{-\bar{\beta}\left(t ; t_{f}\right)}+\mathcal{U}_{c}\left(0^{+}\right) \int_{t}^{t_{f}} e^{-\bar{\beta}(t ; \tau)} d \tau,
$$

for any $t$ in $\left[0, t_{f}\right]$. Merton [48, Chap. 6] states that for no arbitrage, zero wealth must be an absorbing state.

\section{Portfolio stochastic dynamic programming}

Upon applying stochastic dynamic programming (SDP) to the stochastic optimal control problem posed in the previous section, the PDE of stochastic dynamic programming in Hamiltonian form can be shown to be

$$
0=J_{t}^{*}(w, v, t)+\mathcal{H}\left(w, v, t ; u^{*}(w, v, t), c^{*}(w, v, t)\right),
$$

where $J_{t}^{*}(w, v, t)$ is the time partial derivative of $J^{*}(w, v, t)$ and the (pseudo) Hamiltonian is

$$
\begin{aligned}
\mathcal{H}(w, v, t ; u, c) \equiv & -\beta(t) J^{*}(w, v, t)+\mathcal{U}_{c}(c)+\left(r(t)+\left(\mu_{s}(t)-r(t)\right) u w-c\right) J_{w}^{*}(w, v, t) \\
+ & \frac{1}{2} v u^{2} J_{w w}^{*}(w, v, t)+\frac{1}{2} \sigma_{v}^{2}(t) v J_{v v}^{*}(w, v, t)+\kappa_{v}(t)\left(\theta_{v}(t)-v\right) J_{v}^{*}(w, v, t) \\
+ & \lambda_{s}(t)\left(\frac{p_{1}(v, t)}{|a|(v, t)} \int_{a(v, t)}^{0}+\frac{p_{2}(v, t)}{b(v, t)} \int_{0}^{b(v, t)}\right) \\
& \cdot\left(J^{*}(K(u, q) w, v, t)-J^{*}(w, v, t)\right) d q,
\end{aligned}
$$

where

$$
K(u, q) \equiv 1+\left(e^{q}-1\right) u
$$

is the critical function for the natural jump bankruptcy condition [59] to guarantee no bankruptcy. (See the applied derivations in Hanson [22, page 190, Exercises 3-4] in the case of discounting.) The double-uniform density (2.2) has been used to obtain the explicit jump-integral formulation in the last line of (3.2). 


\subsection{Wealth jump no-bankruptcy constraint}

The no bankruptcy condition requires that wealth be nonnegative, so this must apply to the prejump wealth, $w \geq 0$, and the postjump wealth, $K(u, q) w \geq 0$. Hence, we must have

$$
K(u, q) \geq 0 .
$$

Since the variate and parameters of the market double-uniform jump-amplitude distribution satisfy $a(v, t) \leq q \leq b(v, t)$ and $a(v, t)<0<b(v, t)$, then the lower bound on the critical function satisfies

$$
K(u, q) \geq\left\{\begin{array}{ll}
K(u, a(v, t)), & u>0 \\
K(u, b(v, t)), & u<0
\end{array}\right\} \geq 0
$$

This leads to the natural jump-bankruptcy, stock-fraction control bounds to enforce the no-bankruptcy condition upon reformulating the lemma in Zhu and Hanson [59] for dependence on stochasticvolatility, $v$, in addition to dependence on time, $t$.

\section{Lemma 3.1 Jump stock-fraction control bounds for non-negative wealth:}

$$
\widehat{u}_{0}^{(\min )}(v, t) \equiv \frac{-1}{\nu_{s}(v, t, b(v, t))}=\frac{-1}{e^{b(v, t)}-1} \leq u \leq \frac{+1}{1-e^{a(v, t)}}=\frac{+1}{-\nu_{s}(v, t, a(v, t))} \equiv \widehat{u}_{0}^{(\max )}(v, t) .
$$

\section{Remarks 3.1:}

- Here, $\left[\widehat{u}_{0}^{(\min )}(v, t), \widehat{u}_{0}^{(\max )}(v, t)\right]$ naturally define the largest admissible stock-fraction control space due to the no bankruptcy wealth constraint. When the mark space, $[a(t), b(t)]$, is finite such that $-B_{a}^{+} \leq a(v, t) \leq-B_{a}^{-}<0<B_{b}^{-} \leq b(v, t) \leq B_{b}^{+}$for some positive constants $B_{a}^{ \pm}$and $B_{b}^{ \pm}$, then $\widehat{u}_{0}^{(\min )}(v, t)$ and $\widehat{u}_{0}^{(\max )}(v, t)$ are obviously finite, since $0<1-e^{-B_{a}^{-}} \leq$ $1-e^{a(v, t)} \leq 1-e^{-B_{a}^{+}}<1$ with similar bounds for the denominator $e^{b(v, t)}-1$.

- However, if the jump distribution is of infinite range like the log-normal and log-double exponential jump-amplitude distribution, then the admissible stock-fraction controls must be in $[0,1]$, and short-selling as well as borrowing would be severely restricted. For the case of diffusion only with stochastic volatility (SVD), this extra restriction does not apply due to the absence of jumps.

\subsection{Hamiltonian regular optimization conditions}

Before attempting to solve the PDE of SDP, the Hamiltonian equations are used to get the critical points that determine the regular controls, i.e., the optimal controls in absence of constraints. Thus, the critical point for regular consumption control is found from

$$
\left(\frac{\partial \mathcal{H}}{\partial c}\right)^{(\mathrm{reg})}\left(w, v, t ; u^{(\mathrm{reg})}, c^{(\mathrm{reg})}\right)=\mathcal{U}_{c}^{\prime}\left(c^{(\mathrm{reg})}(w, v, t)\right)-J_{w}^{*}(w, v, t)=0,
$$

so $c^{(\mathrm{reg})}(w, v, t)$ is given implicitly by

$$
\mathcal{U}_{c}^{\prime}\left(c^{(\mathrm{reg})}(w, v, t)\right)=J_{w}^{*}(w, v, t)
$$

and $c^{*}(w, v, t)=c^{(\mathrm{reg})}(w, v, t)$ if $c^{(\mathrm{reg})}(w, v, t) \leq w \cdot C_{0}^{(\max )}(V(t), t)$. The optimal consumption control will generally be a composite bang-regular-bang control,

$$
c^{*}(w, v, t)=\left\{\begin{array}{ll}
0, & c^{(\mathrm{reg})}(w, v, t) \leq 0 \\
c^{(\mathrm{reg})}(w, v, t), & 0 \leq c^{(\mathrm{reg})}(w, v, t) \leq w \cdot C_{0}^{(\max )}(v, t) \\
w \cdot C_{0}^{(\max )}(v, t), & w \cdot C_{0}^{(\max )}(v, t) \leq c^{(\mathrm{reg})}(w, v, t)
\end{array}\right\} .
$$


The Hamiltonian condition for the regular stock-fraction control is

$$
\begin{aligned}
\left(\frac{\partial \mathcal{H}}{\partial u}\right)^{(\mathrm{reg})}\left(w, v, t ; u^{(\mathrm{reg})}, c^{(\mathrm{reg})}\right)= & \left(\mu_{s}(t)-r(t)\right) w J_{w}^{*}(w, v, t)+v u^{(\mathrm{reg})}(w, v, t) w^{2} J_{w w}^{*}(w, v, t) \\
& +\lambda_{s}(t)\left(\frac{p_{1}(v, t)}{|a|(v, t)} \int_{a(v, t)}^{0}+\frac{p_{2}(v, t)}{b(v, t)} \int_{0}^{b(v, t)}\right) \\
& \cdot\left(e^{q}-1\right) w J_{w}^{*}\left(K\left(u^{(\mathrm{reg})}(w, v, t), q\right) w, v, t\right) d q=0,
\end{aligned}
$$

with sufficient differentiability of $J^{*}$ using (3.3). So, $u^{(\mathrm{reg})}(w, v, t)$ is given implicitly by

$$
\begin{aligned}
v w^{2} J_{w w}^{*}(w, v, t) u^{(\mathrm{reg})}(w, v, t)= & -\left(\mu_{s}(t)-r(t)\right) w J_{w}^{*}(w, v, t) \\
& -\lambda_{s}(t) w\left(\frac{p_{1}(v, t)}{|a|(v, t)} \int_{a(v, t)}^{0}+\frac{p_{2}(v, t)}{b(v, t)} \int_{0}^{b(v, t)}\right) \\
& \cdot\left(e^{q}-1\right) w J_{w}^{*}\left(K\left(u^{(\mathrm{reg})}(w, v, t), q\right) w, v, t\right) d q
\end{aligned}
$$

and $u^{*}(w, v, t)=u^{(\mathrm{reg})}(w, v, t)$ if $u^{(\mathrm{reg})}(w, v, t)$ is an admissible control, assuming that $U(t)$ is an admissible instantaneous stock-fraction control if

$$
U_{0}^{(\min )}(v, t) \leq U(t) \leq U_{0}^{(\max )}(v, t)
$$

assuming specified bounds $U_{0}^{(\min )}(v, t)$ and $U_{0}^{(\max )}(v, t)$, are independent of $w$. Hence, the optimal stock-fraction control will generally be a composite bang-regular-bang control,

$$
u^{*}(w, v, t)=\left\{\begin{array}{ll}
U_{0}^{(\min )}(v, t), & u^{(\mathrm{reg})}(w, v, t) \leq U_{0}^{(\min )}(v, t) \\
u^{(\operatorname{reg})}(w, v, t), & U_{0}^{(\min )}(v, t) \leq u^{(\mathrm{reg})}(w, v, t) \leq U_{0}^{(\max )}(v, t) \\
U_{0}^{(\max )}(v, t), & U_{0}^{(\max )}(v, t) \leq u^{(\mathrm{reg})}(w, v, t)
\end{array}\right\} .
$$

A good choice for the admissible bounds, $U_{0}^{(\min )}(v, t)$ and $U_{0}^{(\max )}(v, t)$, would be the natural stockfraction control jump bounds, $\widehat{u}_{0}^{(\min )}(v, t)$ and $\widehat{u}_{0}^{(\max )}(v, t)$, given in (3.4).

\section{CRRA canonical solution to optimal portfolio problem}

The constant relative risk aversion (CRRA) utility when $\gamma<1$ is a power utility [48], but is a logarithm when the power $\gamma$ is zero,

$$
\mathcal{U}(x)=\left\{\begin{array}{ll}
x^{\gamma} / \gamma, & \gamma \neq 0 \\
\ln (x), & \gamma=0
\end{array}\right\} .
$$

The range $\gamma<1$ represents several kinds of risk aversion. The utility corresponding to the value $\gamma=0$, arising from the well-defined limit of $\left(x^{\gamma}-1\right) / \gamma$ as $\gamma \rightarrow 0$, is a popular level of risk aversion associated with the Kelly capital growth criterion [38]. The negative range $\gamma<0$ represents extreme risk aversion, and the range $0<\gamma<1$ represents a more moderate level of risk aversion. The value $\gamma=1$ signifies risk-neutral behavior and the remainder $\gamma>1$ means risk-loving behavior. 


\subsection{CRRA power case, $\gamma<1$, but $\gamma \neq 0$}

Setting both utilities to a common form, $\mathcal{U}_{c}(x)=\mathcal{U}(x)=\mathcal{U}_{w}(x)$, and noting the final condition (2.9) now is $J^{*}\left(w, v, t_{f}^{-}\right)=\mathcal{U}(w)$, the following CRRA canonical form of the solution is suggested for the SVJD vector process,

$$
J^{*}(w, v, t)=\mathcal{U}(w) J_{0}(v, t),
$$

when $\gamma \neq 0$ and $\gamma<1$, where $J_{0}(v, t)$ is a function of the variance and time that is to be determined based on the consistency of (4.2). The $\gamma=0$ case requires an additional wealth-independent term $J_{1}(v, t)$ and the risk-neutral $\gamma=1$ case leads to a singular control problem. The original final condition (2.9) yields the greatly reduced final condition $J_{0}\left(v, t_{f}\right)=1$. The solution derivative $J_{w}^{*}(w, v, t)=w^{\gamma-1} J_{0}(v, t)$ is valid even when $\gamma=0$ and leads to

$$
\left(c^{(\mathrm{reg})}\right)^{\gamma-1}(w, v, t)=w^{\gamma-1} J_{0}(v, t) .
$$

This can be solved explicitly for the regular consumption control,

$$
c^{(\mathrm{reg})}(w, v, t)=w J_{0}^{1 /(\gamma-1)}(v, t) \equiv w c_{0}^{(\mathrm{reg})}(v, t)
$$

where $c_{0}^{(\mathrm{reg})}(v, t)=J_{0}^{1 /(\gamma-1)}(v, t) \leq C_{0}^{(\max )}(v, t)$ and $0 \leq C_{0}^{(\max )}(v, t) \leq 1$, the fraction of wealth depending on investor preference. Note that the linear form (4.3) in $w$ is consistent with the linear bound (2.7) on the consumption $C(t)$. In the presence of consumption control constraints, the general optimal consumption control $c^{*}(w, v, t)=w \cdot c_{0}^{*}(v, t)$ is calculated from the composite form (3.6) $\operatorname{using} c^{(\mathrm{reg})}(w, v, t)=w \cdot c_{0}^{(\mathrm{reg})}(v, t)$.

Next using $J_{w w}^{*}(w, v, t)=(\gamma-1) w^{\gamma-2} J_{0}(v, t)$ similarly leads to a reduced implicit formula for the regular stock fraction control from (3.7),

$$
u^{(\mathrm{reg})}(w, v, t) \equiv u_{0}^{(\mathrm{reg})}(v, t)=\frac{1}{(1-\gamma) v}\left(\mu_{s}(t)-r(t)+\lambda_{s}(t) I_{1}\left(u_{0}^{(\mathrm{reg})}(v, t), v, t ; \gamma\right)\right),
$$

independent of the wealth $w$, where

$$
I_{1}(u, v, t ; \gamma) \equiv\left(\frac{p_{1}(v, t)}{|a|(v, t)} \int_{a(v, t)}^{0}+\frac{p_{2}(v, t)}{b(v, t)} \int_{0}^{b(v, t)}\right)\left(e^{q}-1\right) K^{\gamma-1}(u, q) d q
$$

is a jump integral. Note that in the pure diffusion CRRA utility case with constant coefficients, i.e., $\mu_{s}(t)=\mu_{0}, r(t)=r_{0}, v=\sigma_{0}^{2}$ and $\lambda_{s}(t)=0$, the regular control in (4.4) becomes Merton's fraction [43],

$$
u^{(\mathrm{reg})}\left(w, \sigma_{0}^{2}, t\right)=\frac{\mu_{0}-r_{0}}{(1-\gamma) \sigma_{0}^{2}}
$$

In the presence of stock-fraction control constraints, the general optimal stock-fraction control

$$
u^{*}(w, v, t)=u_{0}^{*}(v, t)
$$

is calculated from the composite form (3.9) with bounds (3.8) using

$$
u^{(\mathrm{reg})}(w, v, t)=u_{0}^{(\mathrm{reg})}(v, t) .
$$

It is easy to see from (4.4) that

$$
u_{0}^{(\mathrm{reg})}(v, t)=\mathrm{O}(1 / v) \text { as } v \rightarrow 0^{+},
$$


since this implies, for $\gamma-1<0$, asymptotic consistency by

$$
K^{\gamma-1}\left(u_{0}^{(\mathrm{reg})}(v, t), q\right)=\mathrm{O}\left(\left(u_{0}^{(\mathrm{reg})}\right)^{\gamma-1}(v, t)\right)=\mathrm{O}\left(v^{1-\gamma}\right)=\mathrm{o}(1) \text { as } v \rightarrow 0^{+}
$$

Using these reduced control solution forms leads to the CRRA reduced PIDE for SDP after some algebra,

$$
\begin{aligned}
0= & J_{0, t}(v, t)-\beta(t) J_{0}(v, t)+(1-\gamma) J_{0}^{\gamma /(\gamma-1)}(v, t) \\
& +\gamma\left(r(t)+\frac{1}{2(1-\gamma) v}\left(\left(\mu_{s}(t)-r(t)\right)^{2}-\lambda_{s}^{2}(t) I_{1}^{2}\left(u_{0}^{*}(v, t), v, t ; \gamma\right)\right)\right) J_{0}(v, t) \\
& +\lambda_{s}(t)\left(I_{2}\left(u_{0}^{*}(v, t), v, t ; \gamma\right)-1\right) J_{0}(v, t) \\
& +\frac{1}{2} \sigma_{v}^{2}(t) v J_{0, v v}(v, t)+\kappa_{v}(t)\left(\theta_{v}(t)-v\right) J_{0, v}(v, t),
\end{aligned}
$$

where a second jump integral is

$$
I_{2}(u, v, t ; \gamma) \equiv\left(\frac{p_{1}(v, t)}{|a|(v, t)} \int_{a(v, t)}^{0}+\frac{p_{2}(v, t)}{b(v, t)} \int_{0}^{b(v, t)}\right) K^{\gamma}(u, q) d q,
$$

provided $\gamma \neq 0$.

\subsection{CRRA logarithmic (Kelly criterion) case, $\gamma=0$}

In the logarithmic case, the canonical solution is no longer purely linear in the utility $\mathcal{U}(w)$ of wealth as in (4.2) for the power case, but is affine in $\mathcal{U}(w)=\ln (w)$,

$$
J^{*}(w, v, t)=\ln (w) J_{0}(v, t)+J_{1}(v, t),
$$

where $J_{1}(v, t)$ is a parallel solution form arising from partial derivatives of $J(w, v, t)$ with respect to $\ln (w)$. The final condition, $J\left(w, v, t_{f}\right)=\mathcal{U}(w)=\ln (w)$, produces two parallel final conditions, $J_{0}\left(v, t_{f}\right)=1$ and $J_{1}\left(v, t_{f}\right)=0$, since $\ln (w)$ and the constant 1 are independent functions of $w$.

Since the determination of the regular control functions involves only derivatives of $J(w, v, t)$ with respect to wealth $w$, the formulas in (4.3) and (4.4) are valid for $\gamma=0$. So

$$
c^{(\mathrm{reg})}(w, v, t) \equiv w c_{0}^{(\mathrm{reg})}(v, t)=w / J_{0}(v, t)
$$

and

$$
u^{(\mathrm{reg})}(w, v, t) \equiv u_{0}^{(\mathrm{reg})}(v, t)=\frac{1}{v}\left(\mu_{s}(t)-r(t)+\lambda_{s}(t) I_{1}\left(u_{0}^{(\mathrm{reg})}(v, t), v, t ; 0\right)\right) .
$$

However, the reduced SDP PIDE is not the same as in (4.8) when $\gamma \neq 0$. Two parallel reduced PIDEs are obtained. The first is found by separately equating the cumulative coefficient of $\ln (w)$ to zero by independence, yielding a linear PIDE in $J_{0}(v, t)$,

$$
0=J_{0, t}(v, t)-\beta(t) J_{0}(v, t)+1+\frac{1}{2} \sigma_{v}^{2}(t) v J_{0, v v}(v, t)+\kappa_{v}(t)\left(\theta_{v}(t)-v\right) J_{0, v}(v, t) .
$$

The second for the remaining terms yields another linear PIDE, but in $J_{1}(v, t)$,

$$
\begin{aligned}
0= & J_{1, t}(v, t)+(r(t)-\beta(t)) J_{1}(v, t)-\ln \left(J_{0}(v, t)\right)-1 \\
& +\left(\frac{1}{2 v}\left(\left(\mu_{s}(t)-r(t)\right)^{2}-\lambda_{s}^{2}(t) I_{1}^{2}\left(u_{0}^{*}(v, t), v, t ; 0\right)\right)+\lambda_{s}(t) I_{2}\left(u_{0}^{*}(v, t), v, t ; 0\right)\right) J_{0}(v, t)(4 . \\
& +\frac{1}{2} \sigma_{v}^{2}(t) v J_{1, v v}(v, t)+\kappa_{v}(t)\left(\theta_{v}(t)-v\right) J_{1, v}(v, t),
\end{aligned}
$$


where

$$
I_{2}(u, v, t ; 0) \equiv\left(\frac{p_{1}(v, t)}{|a|(v, t)} \int_{a(v, t)}^{0}+\frac{p_{2}(v, t)}{b(v, t)} \int_{0}^{b(v, t)}\right) \ln (K(u, q)) d q,
$$

in this special case. Note that the parallel PIDEs are uni-directionally coupled, so that if (4.11) for $J_{0}(v, t)$ is solved first, then (4.12) for $J_{1}(v, t)$ can be solved as a single PIDE using the solution $J_{0}(v, t)$.

The static case of logarithmic utility of wealth or Kelly criterion is surveyed by MacLean and Ziemba [42]. They note that several legendary investors have used the Kelly criterion. One is Edward O. Thorp who was a prime promoter of the criterion in gambling and market investments. Another is Warren Buffet, who is identified as a Kelly criterion investor from the performance of the BerkshireHathaway fund.

\subsection{Transformation to an implicit type of Bernoulli equation}

In the pure stochastic diffusion case with constant coefficients, the PDE of SDP becomes a Bernoulli ODE in time using the CRRA power utility [43, 44]. Using the classical Bernoulli transformation, the nonlinear ODE can be transformed to a linear ODE suitable for very standard methods. In the stochastic jump-diffusion case with time dependent coefficients and control constraints, the PDE of SDP becomes a Bernoulli ODE complicated by implicit dependence through the jump integrals and optimal controls $[23,25,27,28,29,59]$. The Bernoulli transformation still has significant benefits for the case $\gamma<1$ and $\gamma \neq 0$, but additional iterations are needed to treat the implicit dependencies. In the SVJD case, the stochastic volatility terms mean that the PDE of SDP remains a PDE, but with some Bernoulli nonlinear properties that can be reduced to something simpler.

First, the coefficients of $J_{0}(v, t)$ are collected together, excluding a consumption term, in the following form,

$$
\begin{aligned}
g_{1}(v, t ; \gamma) \equiv & \frac{1}{1-\gamma}\left(-\beta(t)+\gamma\left(r(t)+\left(\mu_{s}(t)-r(t)\right) u_{0}^{*}(v, t)\right)+\frac{\gamma}{2} v\left(u_{0}^{*}\right)^{2}(v, t)\right. \\
& \left.+\frac{\lambda_{s}(t)}{1-\gamma}\left(I_{2}\left(u_{0}^{*}(v, t), t\right)-1\right)\right) .
\end{aligned}
$$

Next, the two optimal consumption terms are collected in a common coefficient for the nonlinear Bernoulli term,

$$
g_{2}(v, t ; \gamma) \equiv\left(\left(\frac{c_{0}^{*}(v, t)}{c_{0}^{(\mathrm{reg})}(v, t)}\right)^{\gamma}-\gamma\left(\frac{c_{0}^{*}(v, t)}{c_{0}^{(\mathrm{reg})}(v, t)}\right)\right)
$$

where the following identity has been used,

$$
\left(c_{0}^{*}\right)^{\gamma}(v, t)-\gamma c_{0}^{*}(v, t) J_{0}(v, t) \equiv \frac{g_{2}(v, t ; \gamma)}{1-\gamma} J_{0}^{\gamma /(\gamma-1)}(v, t) .
$$

Finally, a Bernoulli-like PDE is obtained,

$$
\begin{aligned}
0= & J_{0, t}(v, t)+(1-\gamma)\left(g_{1}(v, t ; \gamma) J_{0}(v, t)+g_{2}(v, t ; \gamma) J_{0}^{\gamma /(\gamma-1)}(v, t)\right) \\
& +\frac{1}{2} \sigma_{v}^{2}(t) v J_{0, v v}(v, t)+\kappa_{v}(t)\left(\theta_{v}(t)-v\right) J_{0, v}(v, t) .
\end{aligned}
$$


This is a nonlinear diffusion equation with implicit coupling to $c_{0}^{*}(v, t), c_{0}^{(\mathrm{reg})}(v, t)$ and $u_{0}^{*}(v, t)$.

For the problem in (4.16), the simplifying Bernoulli transformation is

$$
y(v, t)=J_{0}^{1 /(1-\gamma)}(v, t)
$$

with inverse

$$
J_{0}(v, t)=y^{1-\gamma}(v, t)
$$

and the transformed PDE, having almost all linear terms without counting the implicit control and variance dependence, is

$$
\begin{aligned}
0= & y_{t}(v, t)+g_{1}(v, t ; \gamma) y(v, t)+g_{2}(v, t ; \gamma) \\
& +\frac{1}{2} \sigma_{v}^{2}(t) v y_{v v}(v, t)+\left(\kappa_{v}(t)\left(\theta_{v}(t)-v\right) y_{v}(v, t)-\frac{\gamma \sigma_{v}^{2}(t) v}{y(v, t)}\right),
\end{aligned}
$$

assuming $y(v, t) \neq 0$ and with final condition $y\left(v, t_{f}\right)=1$. It can be seen from (3.7) that the regular stock-fraction control $u_{0}^{(\mathrm{reg})}(v, t)$ becomes unbounded as the volatility $v \rightarrow 0^{+}$, which should be handled by a finite control space $\left[U_{0}^{(\min )}(v, t), U_{0}^{(\max )}(v, t)\right]$ as indicated by the jump-bankruptcy bounds $\left[\widehat{u}_{0}^{(\min )}(v, t), \widehat{u}_{0}^{(\max )}(v, t)\right]$.

Since the PDE (4.18) can be solved by computational iteration at each time step, (4.18) can be treated like an ODE in time by formally writing the transformed solution in quadratures using an integrating factor,

$$
y(v, t)=e^{\bar{g}_{1}\left(v, t ; t_{f} ; \gamma\right)}+\int_{t}^{t_{f}} e^{\bar{g}_{1}(v, t ; \tau ; \gamma)} \widehat{G}_{2}(v, \tau ; \gamma) d \tau
$$

where

$$
\bar{g}_{1}(v, t ; s ; \gamma) \equiv \int_{t}^{s} g_{1}(v, y ; \gamma) d y
$$

and

$$
\widehat{G}_{2}(v, t ; \gamma) \equiv g_{2}(v, t ; \gamma)+\frac{1}{2} \sigma_{v}^{2}(t) v y_{v v}(v, t)+\left(\kappa_{v}(t)\left(\theta_{v}(t)-v\right) y_{v}(v, t)-\frac{\gamma \sigma_{v}^{2}(t) v}{y(v, t)}\right)
$$

The latter definition (4.21) includes the suppressed variance-derivative and consumption terms that can be treated by iteration. Thus, the implicit solution for the variance-time function can be written as

$$
J_{0}(v, t)=\left(e^{\bar{g}_{1}\left(v, t ; t_{f} ; \gamma\right)}+\int_{t}^{t_{f}} e^{\bar{g}_{1}(v, t ; \tau ; \gamma)} \widehat{G}_{2}(v, \tau ; \gamma) d \tau\right)^{1-\gamma}
$$

with the full wealth-dependent solution given by

$$
J^{*}(w, v, t)=\frac{w^{\gamma}}{\gamma} J_{0}(v, t)
$$




\subsubsection{CRRA logarithmic case formal solution, $\gamma=0$}

For the $\gamma=0$ case, the Bernoulli transformation (4.17) is the identity operator. So both solution forms $J_{0}(v, t)$ and $J_{1}(v, t)$ satisfy unidirectionally coupled linear equations that are solved in sequence. As for the general risk-averse case, the PIDEs (4.11) and (4.12) are prepared for better-posed timestepping iterations using integrating factors, so that for the coefficient of $\ln (w)$,

$$
J_{0}(v, t)=e^{-\bar{\beta}\left(t ; t_{f}\right)}+\int_{t}^{t_{f}} e^{-\bar{\beta}(t ; \tau)} \widehat{G}_{0}(v, \tau) d \tau
$$

since $J_{0}\left(v, t_{f}\right)=1$, where

$$
\widehat{G}_{0}(v, t) \equiv 1+\frac{1}{2} \sigma_{v}^{2}(t) v J_{0, v v}(v, t)+\kappa_{v}(t)\left(\theta_{v}(t)-v\right) J_{0, v}(v, t)
$$

includes the variance-derivative terms. Given $J_{0}(v, t)$, the wealth-independent term satisfies

$$
J_{1}(v, t)=\int_{t}^{t_{f}} e^{\bar{r}(t ; \tau)-\bar{\beta}(t ; \tau)} \widehat{G}_{1}(v, \tau) d \tau,
$$

since $J_{1}\left(v, t_{f}\right)=0$, where

$$
\bar{r}(t ; \tau) \equiv \int_{t}^{\tau} r(y) d y
$$

and

$$
\begin{aligned}
\widehat{G}_{1}(v, t) \equiv & -\ln \left(J_{0}(v, t)\right)-1+\frac{1}{2 v}\left(\left(\mu_{s}(t)-r(t)\right)^{2}-\lambda_{s}^{2}(t) I_{1}^{2}\left(u_{0}^{*}(v, t), v, t ; 0\right)\right) J_{0}(v, t) \\
& +\lambda_{s}(t) I_{2}\left(u_{0}^{*}(v, t), v, t ; 0\right) J_{0}(v, t) \\
& +\frac{1}{2} \sigma_{v}^{2}(t) v J_{1, v v}(v, t)+\kappa_{v}(t)\left(\theta_{v}(t)-v\right) J_{1, v}(v, t),
\end{aligned}
$$

which includes suppressed $J_{0}$ and $J_{1}$-variance-derivative terms. In summary, for $\gamma=0$,

$$
J^{*}(w, v, t)=\ln (w) J_{0}(v, t)+J_{1}(v, t) .
$$

\section{Optimal wealth trajectory without bankruptcy}

To check whether the no bankruptcy condition $W(t) \geq 0$ holds, the optimal controls for the stockfraction (4.4) and consumption (4.3) are substituted into the wealth SDE (2.6) obtaining a geometric jump-diffusion,

$$
d W^{*}(t)=W^{*}(t)\left(\mu_{W}^{*}(V(t), t) d t+\sqrt{V(t)} d G_{s}(t)+\left(e^{Q}-1\right) d P_{s}(t ; Q)\right),
$$

coupled with the stochastic volatility SDE (2.4), where

$$
\mu_{W}^{*}(v, t) \equiv r(t)+\left(\mu_{s}(t)-r(t)\right) u_{0}^{*}(v, t)-c_{0}^{*}(v, t) .
$$

An exponential form of the solution for (5.1) can be found by (1) using the standard logarithmic transform $L(t)=\ln \left(W^{*}(t)\right)$ for the geometric jump-diffusion (5.1), (2) using the corresponding SVJD 
extension of Itô's stochastic chain rule to remove $W^{*}(t)$ from the right-hand-side (see Hanson [22]), and (3) integrating the simplified SDE, yielding

$$
W^{*}(t)=W_{0} \exp \left(\int_{o}^{t}\left(\mu_{L}^{*}(V(\tau), \tau) d \tau+\sqrt{V(\tau)} d G_{s}(\tau)+Q d P_{s}(\tau ; Q)\right)\right),
$$

where

$$
\mu_{L}^{*}(v, t) \equiv \mu_{W}^{*}(v, t)-v / 2 .
$$

Assumptions 5.0: All relevant coefficients, i.e.,

$$
\begin{aligned}
& S_{0}, \mu_{s}(t), \lambda_{s}(t), \nu_{s}(v, t, q), a(v, t), b(v, t), p_{1}(v, t), p_{2}(v, t), V_{0}, \kappa_{v}(t), \theta_{v}(t), \sigma_{v}(t), \\
& B_{0}(t), r(t), W_{0}, U_{s}(t), C(t), C_{0}^{(\max )}(v, t), \beta(t), U_{0}^{(\max )}(v, t), U_{0}^{(\min )}(v, t) \text { and } \gamma,
\end{aligned}
$$

are assumed to be bounded.

In particular, the practical bounds on the Gaussian noise are

$$
\left|G_{s}(t)\right| \leq B_{G} t \quad \& \quad\left|G_{v}(t)\right| \leq B_{G} t
$$

for a large finite, positive constant $B_{G}$ and finite horizon $t \leq T$.

The bounds on $a(v, t), b(v, t), p_{1}(v, t), p_{2}(v, t)$ and $C_{0}^{(\max )}(v, t)$ have already been stated. Both $U_{0}^{(\max )}(v, t)$ and $U_{0}^{(\min )}(v, t)$ have been superseded by the jump forced stock-fraction control bounds in (3.4), $\widehat{u}_{0}^{(\min )}(v, t)$ and $\widehat{u}_{0}^{(\max )}(v, t)$, respectively.

Since $W_{0}>0$ has been assumed for the initial condition, we have, using (5.2) when $\gamma<1$ and $\gamma \neq 0$, the following lemma.

Lemma 5.0 Non-negativity of optimal wealth trajectory: Under the bounded coefficients assumptions and the practical bounds (5.3), then

$$
W(t)>0
$$

Practical Remarks 5.0: In particular, we assume that the Gaussian processes are for all practical purposes bounded, i.e., $\left|G_{s}(t)\right| \leq B_{G} t$ and $\left|G_{v}(t)\right| \leq B_{G} t$, since in real markets the noise is bounded and the usual assumption of unbounded noise is only an artifact of the ideal mathematical models of Wiener or Brownian motion. The bounds (5.3) mean that the Gaussian extremes of very small probability are not realistic. It does not make sense for practical purposes to spend time examining the importance, if any, of the most extreme deviations with the most small probabilities. There are also the circuit breakers [3] of the NYSE that prevent, in installments, the most extreme market changes like those in 1987. Again, note that the reasons for and consequently the results in (5.4) are quite different from those in [37] and [51] for pure diffusions. Real markets have extremes, but they are bounded extremes.

Thus, Lemma 5.0 shows there is no possibility of bankruptcy or zero wealth starting from positive initial wealth for the CRRA power utility with $\gamma<1$, including $\gamma=0$.

\section{Verification of nonnegativity of stochastic variance}

Note that there could be a potential serious problem with the optimal stock-fraction control $u_{0}^{*}(v, t)$ due to its dependence on the regular stock-fraction control (4.4),

$$
u^{(\mathrm{reg})}(w, v, t) \equiv u_{0}^{(\mathrm{reg})}(v, t)=\frac{\left(\mu_{s}(t)-r(t)\right)}{(1-\gamma) v}+\frac{\lambda_{s}(t)}{(1-\gamma) v} I_{1}\left(u_{0}^{(\mathrm{reg})}(v, t), v, t\right)
$$


which is singular when either $v \rightarrow 0^{+}$or $\gamma \rightarrow 1^{-}$, but the finite bounds on $u_{0}^{*}(v, t)$ provide a cutoff for these singularities. In addition, the practical Gaussian bounds (5.3) imply that $0 \leq V(t) \leq B_{V}$, for some positive constant $B_{V}$ and for all practical purposes.

On the other hand, the nonnegativity of the stochastic variance, $V(t) \geq 0$, was settled long ago for the square-root diffusion model by Feller [17] using very elaborate Laplace transform techniques on the corresponding Kolmogorov forward equation to obtain the noncentral chi-squared distribution for the process. He has given the boundary condition classification for the process in terms of the parameters, which helps to determine which values would guarantee positivity preservation in the range of nonnegativity preserving values. So, in the time-independent form notation here, positivity is assured if $1<2 \kappa_{v} \theta_{v} / \sigma_{v}^{2}$ with zero boundary conditions in value and flux, while if $0<2 \kappa_{v} \theta_{v} / \sigma_{v}^{2}<1$ then only nonnegativity can be assured. See Cox et al. [13], Glassman [20], Jäckel [33], Broadie and Kaya [11], and Lord et al. [41] for other qualifications and information, including various distribution simulation techniques.

\subsection{Transformation to perfect-square form}

Using the general transformation techniques in Hanson [22] with $Y(t)=F(V(t), t)$, it is possible to find a general perfect square solution to (2.4). Using Itô's lemma, the following transformed SDE is obtained,

$$
d Y(t)=F_{, t}(V(t), t) d t+F_{, v}(V(t), t) d V(t)+\frac{1}{2} F_{, v v}(V(t), t) \sigma_{v}^{2}(t) V(t) d t
$$

to $d t$-precision. Then a simpler form is sought with volatility-independent noise term, i.e.,

$$
d Y(t)=\left(\mu_{y}^{(0)}(t)+\mu_{y}^{(1)}(t) / \sqrt{V(t)}\right) d t+\sigma_{y}(t) d G_{v}(t)
$$

with $Y(0)=F\left(V_{0}, 0\right)$, where $\mu_{y}^{(0)}(t), \mu_{y}^{(1)}(t)$ and $\sigma_{y}(t)$ are time-dependent coefficients to be determined. Equating the coefficients of $d G_{v}(t)$ terms between (6.1) and (6.2), given $V(t)=v>0$, leads to

$$
F_{, v}(v, t)=\left(\frac{\sigma_{y}}{\sigma_{v}}\right)(t) \frac{1}{\sqrt{v}}
$$

and then partially integrating (6.3) yields

$$
F(v, t)=\left(\frac{\sigma_{y}}{\sigma_{v}}\right)(t) \sqrt{v}+c_{1}(t)
$$

which is the desired transformation with a function of integration $c_{1}(t)$. Additional differentiations of (6.3) produce

$$
F_{, t}(v, t)=\left(\frac{\sigma_{y}}{\sigma_{v}}\right)^{\prime}(t) \sqrt{v}+c_{1}^{\prime}(t) \quad \& \quad F_{, v v}(v, t)=-\frac{1}{2}\left(\frac{\sigma_{y}}{\sigma_{v}}\right)(t) v^{-3 / 2} .
$$

Terms of order $v^{0} d t$ imply that $c_{1}^{\prime}(t)=\mu_{y}^{(0)}(t)$, but this equates two unknown coefficients, so we set $\mu_{y}^{(0)}(t)=0$ for simplicity. Equating terms of order $\sqrt{v} d t$ and integrating imply

$$
\left(2\left(\frac{\sigma_{y}}{\sigma_{v}}\right)^{\prime}-\kappa_{v}\left(\frac{\sigma_{y}}{\sigma_{v}}\right)\right)(t)=0 \Longrightarrow\left(\frac{\sigma_{y}}{\sigma_{v}}\right)(t)=\left(\frac{\sigma_{y}}{\sigma_{v}}\right)(0) e^{\bar{\kappa}_{v}(0, t) / 2},
$$


where

$$
\bar{\kappa}_{v}(\tau, t) \equiv \int_{\tau}^{t} \kappa_{v}(y) d y .
$$

For convenience, we set $\sigma_{y}(0)=\sigma_{v}(0)$. For order $v^{-1 / 2} d t$, we obtain

$$
\mu_{y}^{(1)}(t)=e^{\bar{\kappa}_{v}(0, t) / 2}\left(\kappa_{v} \theta_{v}-\frac{1}{4} \sigma_{v}^{2}\right)(t)
$$

completing the coefficient determination.

Assembling these results we form the solution as follows,

$$
Y(t)=2 e^{\bar{\kappa}_{v}(0, t) / 2} \sqrt{V(t)}
$$

and inverting this yields the desired nonnegativity result:

$$
V(t)=e^{-\bar{\kappa}_{v}(0, t)}\left(\frac{Y(t)}{2}\right)^{2} \geq 0,
$$

due to the perfect square form, where

$$
Y(t)=2 \sqrt{V_{0}}+\int_{0}^{t} e^{\bar{\kappa}_{v}(0, s) / 2}\left(\left(\frac{\kappa_{v} \theta_{v}-\frac{1}{4} \sigma_{v}^{2}}{\sqrt{V}}\right)(s) d s+\left(\sigma_{v} d G_{v}\right)(s)\right) .
$$

This is an implicit form that is singular unless the solution $V(t)$ is bounded away from zero, $V(t)>0$. More generally it is desired that the solution is such that $1 / \sqrt{V(t)}$ is integrable in $t$ as $V(t) \rightarrow 0^{+}$, so the singularity will be ignorable in theory.

\subsection{Proper singular limit formulation suitable for computation}

However, as $V(t) \rightarrow 0^{+}$, the validity of neglecting higher order terms in the Taylor expansion underlying Itô's lemma is questionable, unless the integral is treated as a singular integral and the method of integration steps is properly specified.

First (6.5)-(6.6) are simply reformulated as

$$
V(t)=e^{-\bar{\kappa}_{v}(0, t)}\left(\sqrt{V_{0}}+\frac{1}{2} I_{g}(t)\right)^{2},
$$

where

$$
I_{g}(t)=\int_{0}^{t} e^{\bar{\kappa}_{v}(0, s) / 2}\left(\left(\frac{\kappa_{v} \theta_{v}-\frac{1}{4} \sigma_{v}^{2}}{\sqrt{V}}\right)(s) d s+\left(\sigma_{v} d G_{v}\right)(s)\right) .
$$

Modifying the method of ignoring the singularity [14] to this implicit singular formulation, let

$$
V^{\left(\varepsilon_{v}\right)}(t)=\max \left(V(t), \varepsilon_{v}\right)
$$

where $\varepsilon_{v}>0$ such that $\Delta t / \sqrt{\varepsilon_{v}} \ll 1$ as some reference numerical increment $\Delta t \rightarrow 0^{+}$to ensure that the time-step goes to zero faster than the cutoff singular square root denominator. Next (6.7)-(6.8) 
is reformulated as a recursion using some algebra for the next time increment $\Delta t$ and the method of integration is specified for each subsequent time step, i.e.,

$$
V(t+\Delta t)=e^{-\Delta \bar{\kappa}_{v}(0, t)} \lim _{\varepsilon_{v} \rightarrow 0^{+}}\left(\sqrt{V(t)}+\frac{1}{2} e^{-\bar{\kappa}_{v}(0, t) / 2} \Delta I_{g}^{\left(\varepsilon_{v}\right)}(t)\right)^{2},
$$

where

$$
\Delta \bar{\kappa}_{v}(0, t) \equiv \int_{t}^{t+\Delta t} \kappa_{v}(s) d s \rightarrow \kappa_{v}(t) \Delta t
$$

as $\Delta t \rightarrow 0^{+}$. Similarly, a scaled increment of an integral is defined by

$$
\begin{aligned}
e^{-\bar{\kappa}_{v}(0, t) / 2} \Delta I^{\left(\varepsilon_{v}\right)}(t) & \equiv \int_{t}^{t+\Delta t} e^{-\bar{\kappa}_{v}(s, t) / 2}\left(\left(\frac{\kappa_{v} \theta_{v}-\frac{1}{4} \sigma_{v}^{2}}{\sqrt{V^{\left(\varepsilon_{v}\right)}}}\right)(s) d s+\left(\sigma_{v} d G_{v}\right)(s)\right) \\
& \rightarrow\left(\left(\frac{\kappa_{v} \theta_{v}-\frac{1}{4} \sigma_{v}^{2}}{\sqrt{V}}\right)(t) d t+\left(\sigma_{v} d G_{v}\right)(t)\right)
\end{aligned}
$$

such that $\Delta t / \sqrt{\varepsilon_{v}} \rightarrow 0^{+}$as $\Delta t \rightarrow 0^{+}$. An Itô-Taylor expansion to precison $d t$ or small $\Delta t$ confirms that (6.9)-(6.10) yields the Heston [21] model, proving solution consistency. Thus, the square in (6.9) formally justifies the nonnegativity of the variance and the volatility of the Heston [21] model, for a proper computational nonnegativity-preserving procedure.

Note that the zero volatility limit is not a serious concern since the control constraints also provide a cutoff for the volatility. Further, the logarithmic transformation used for the geometric Brownian motion leads to singular derivatives of all orders, but the singularities are exactly cancelled out by the linear property of the underlying SDE.

\subsection{Nonsingular, explicit, exact solution}

In any event, the singular term in (6.7)-(6.8) vanishes in the special parameter case, such that

$$
\kappa_{v}(t) \theta_{v}(t)=\frac{1}{4} \sigma_{v}^{2}(t), \quad \forall t
$$

Hence, we obtain a nonnegative, nonsingular exact solution

$$
V(t)=e^{-\bar{\kappa}_{v}(0, t)}\left(\sqrt{V_{0}}+0.5 \int_{0}^{t} e^{\bar{\kappa}_{v}(0, s) / 2}\left(\sigma_{v} d G_{v}\right)(s)\right)^{2}
$$

with the numerical form corresponding to (6.9)-(6.10),

$$
V(t+\Delta t)=e^{-\Delta \bar{\kappa}_{v}(0, t)}\left(\sqrt{V(t)}+\frac{1}{2} \int_{t}^{t+\Delta t} e^{-\bar{\kappa}_{v}(s, t) / 2}\left(\sigma_{v} d G_{v}\right)(s)\right)^{2} .
$$

Similarly, the chain rule for the integrating factor form $\exp \left(\bar{\kappa}_{v}(0, t)\right) V(t)$ for the stochastic volatility (2.4) leads to a somewhat simpler integrated form,

$$
V(t)=\max \left(V^{(\mathrm{det})}(t)+\int_{0}^{t} e^{-\bar{\kappa}_{v}(s, t)}\left(\sigma_{v} \sqrt{V} d G_{v}\right)(s), 0\right),
$$


using the maximum with respect to zero to remove spurious numerical simulations in absence of a perfect square form. In (6.13),

$$
V^{(\operatorname{det})}(t)=V_{0} e^{-\bar{\kappa}_{v}(0, t)}+\theta_{v}(t)\left(1-e^{-\bar{\kappa}_{v}(0, t)}\right)
$$

is the deterministic part of $V(t)$. Note that there is only a linear change of dependent variable according to the stochastic chain rule [22] using the transformation $Y(t)=\exp \left(\bar{\kappa}_{v}(0, t)\right) V(t)$. So the deterministic part is easily separated out from the square-root dependence and replaces the meanreverting drift term. The $V^{(\mathrm{det})}(t)$ will be positive for positive parameters.

However, as Lord et al. [41] point out, a sufficiently accurate simulation scheme and a large number of simulation nodes are required so that the right-hand side of (2.4) generates nonnegative values. Nonnegative values using the stochastic Euler simulation have been verified for Heston's [21] constant risk-neutralized parameter values $\left(\kappa_{v}=2.00, \theta_{v}=0.01, \sigma_{v}=0.10\right)$ as long as the scaled number of nodes per unit time $N /\left(\kappa_{v} t_{f}\right)>100$.

Hence, since the variance by definition for real processes cannot be negative, practical considerations suggest replacing occurrences of $V(t)$ by $\max (V(t), \epsilon)$, where $\epsilon$ is some numerically small, positive quantity for numerical purposes to account for the appearances of negative variance values.

\section{Conclusions}

The optimal portfolio and consumption problem has been extended to stochastic-volatility, jumpdiffusion environments with the log-double-uniform jump-amplitude distribution. The jump-wealth, no-bankruptcy condition has been reconfirmed with extra benefits due to the natural stock-fraction jump constraints. The constraints help avoid stochastic-volatility and CRRA power exponent singularities in the wealth solution. For all practical purposes the wealth is not just non-negative but also remains positive due to the geometric nature of the wealth process and the constraint singularity protection if the initial wealth is positive.

There are some new, formal results for the nonnegativity of the variance for the Heston [21] model, an implicit perfect square solution in the general parameter case and an explicit form for the case where the speed of reversion times the level of reversion is one quarter of the square of the volatility of the volatility coefficient.

Future directions are to convert jump-diffusion optimal portfolio problem computations to produce SVJD computations.

\section{Acknowledgement}

The author is grateful to Phelim P. Boyle for bringing to his attention the Lord et al. [41] discussion paper comparing simulations of stochastic volatility models along with background.

\section{References}

[1] Y. Aït-Sahalia, "Disentangling Diffusion from Jumps," J. Fin. Econ., vol. 74, 2004, pp. 487528.

[2] T. G. Andersen, L. Benzoni and J. Lund, "An Empirical Investigation of Continuous-Time Equity Return Models,” J. Fin., vol. 57, 2002, pp. 1239-1284. 
[3] C. A. Aourir , D. Okuyama, C. Lott and C. Eglinton, Exchanges - Circuit Breakers, Curbs, and Other Trading Restrictions, 2002, http:// invest-faq.com/articles/ exch-circuit-brkr.html.

[4] G. Bakshi, C. Cao and Z. Chen, "Empirical Performance of Alternative Option Pricing Models," J. Fin., vol. 52, 1997, pp. 2003-2049.

[5] C. A. Ball, and A. Roma, "Stochastic Volatility Option Pricing," J. Fin. and Quant. Anal., vol. 29 (4), 1994, pp. 589-607.

[6] C. A. Ball, and W. N. Torous, "The Maximum Likelihood Esimation of Security Price Volatility: Theory, Evidence, and Application to Option Pricing," J. Bus., vol. 57 (1), 1984, pp. 97-112.

[7] C. A. Ball, and W. N. Torous, "On Jumps in Common Stock Prices and Their Impact on Call Option Prices," J. Fin., vol. 40 (1), 1985, pp. 155-173.

[8] D. Bates,"The Crash of '87: Was It Expected? The Evidence from Option Markets," J. Fin., vol. 46, 1991, pp. 1009-1044.

[9] D. Bates, "Jump and Stochastic Volatility: Exchange Rate Processes Implict in Deutsche Mark in Options," Rev. Fin. Studies, vol. 9, 1996, pp. 69-107.

[10] F. Black and M. Scholes, "The Pricing of Options and Corporate Liabilities," J. Political Economy, vol. 81, 1973, pp. 637-659.

[11] M. Broadie and Ö. Kaya, "Exact Simulation of Stochastic Volatility and Other Affine Jump Diffusion Processes," Oper. Res., vol. 54 (2), 2006, pp. 217-231.

[12] J. C. Cox, J. E. Ingersoll and S. A. Ross, "An Intertemporal General Equilibrium Model of Asset Prices," Econometrica, vol. 53 (2), 1985, pp. 363-384.

[13] J. C. Cox, J. E. Ingersoll and S. A. Ross, "A Theory of the Term Structure of Interest Rates," Econometrica, vol. 53 (2), 1985, pp. 385-408.

[14] P. J. Davis and P. Rabinowitz, "Ignoring the Singularity in Approximate Integration," J. SIAM Num. Anal., vol. 2 (3), 1965, pp. 367-383.

[15] D. Duffie, J. Pan and K. Singleton, "Transform Analysis and Asset Pricing for Affine JumpDiffusions," Econometrica, vol. 68, 2000, pp. 1343-1376.

[16] M. Evans, N. Hastings, and B. Peacock, Statistical Distributions, 3rd edn., John Wiley, New York, NY, 2000.

[17] W. Feller, “Two Singular Diffusion Problems” Ann. Math., vol. 54, 1951, pp. 173-182.

[18] J.-P. Fouque, G. Papanicolaou, and K. R. Sircar, Derivatives in Financial Markets with Stochastic Volatility, Cambridge University Press, Cambridge, UK, 2000.

[19] M. B. Garman and M. J. Klass, "On the Estimation of Security Price Volatilities from Historical Data,” J. Bus., vol. 53 (1), 1980, pp. 67-77.

[20] P. Glasserman, Monte Carlo Methods in Financial Engineering, Springer-Verlag, New York, NY, 2003. 
[21] S. L. Heston, A Closed-form Solution for Options with Stochastic Volatility with Applications to Bond and Currency Options, Rev. Fin. Studies, vol. 6, 1993, pp. 327-343.

[22] F. B. Hanson, Applied Stochastic Processes and Control for Jump-Diffusions: Modeling, Analysis and Computation, Series in Advances in Design and Control, vol. DC13, SIAM Books, Philadelphia, PA, 2007.

[23] F. B. Hanson and J. J. Westman, "Optimal Consumption and Portfolio Policies for Important Jump Events: Modeling and Computational Considerations," Proc. 2001 American Control Conf., 2001, pp. 4556-4561.

[24] F. B. Hanson and J. J. Westman, "Stochastic Analysis of Jump-Diffusions for Financial LogReturn Processes," Proc. Stochastic Theory and Control Workshop, Lecture Notes in Control and Information Sciences, vol. 280, B. Pasik-Duncan (Editor), Springer-Verlag, New York, NY, 2002, pp. 169-184.

[25] F. B. Hanson and J. J. Westman, "Optimal Consumption and Portfolio Control for JumpDiffusion Stock Process with Log-Normal Jumps," Proc. 2002 American Control Conf., 2002, pp. 4256-4261; corrected paper: ftp://ftp.math.uic.edu/pub/Hanson/ACC02/ acc02webcor.pdf.

[26] F. B. Hanson and J. J. Westman, "Jump-Diffusion Stock Return Models in Finance: Stochastic Process Density with Uniform-Jump Amplitude," Proc. 15th Int. Symp. on Mathematical Theory of Networks and Systems, 2002, 7 CD pages.

[27] F. B. Hanson and J. J. Westman, "Computational Methods for Portfolio and Consumption Optimization in Log-Normal Diffusion, Log-Uniform Jump Environments," Proc. 15th Int. Symp. on Mathematical Theory of Networks and Systems, 2002, 9 CD pages.

[28] F. B. Hanson and J. J. Westman, "Portfolio Optimization with Jump-Diffusions: Estimation of Time-Dependent Parameters and Application," Proc. 41st Conf. on Decision and Control, 2002, pp. 377-382; partially corrected paper: ftp://ftp.math.uic.edu/pub/ Hanson/CDC02/cdc02web.pdf .

[29] F. B. Hanson and J. J. Westman, "Optimal Portfolio and Consumption Policies Subject to Rishel's Important Jump Events Model: Computational Methods," Trans. Automatic Control, vol. 48 (3), Special Issue on Stochastic Control Methods in Financial Engineering, 2004, pp. $326-337$.

[30] F. B. Hanson and G. Yan, "American Put Option Pricing for Stochastic-Volatility, JumpDiffusion Models," Proc. 2007 American Control Conf., 2007, pp. 384-389.

[31] J. C. Hull, Options, Futures, \& Other Derivatives, 6th Edition, Prentice-Hall, Englewood Cliffs, NJ, 2006.

[32] J. C. Hull, and A. White, "The Pricing of Options on Assets with Stochastic Volatilities" J. Fin., vol. 42 (2), 1987, pp. 281-300.

[33] P. Jäckel, "Stochastic Volatility Models: Past Present And Future," The Best of Wilmott I: Incorporating the Quatitative Finance Review, P. Wilmott (Editor), John Wiley, New York, NY, 2004, pp. 379-3390. 
[34] R. A. Jarrow and E. R. Rosenfeld, "Jump Risks and the Intertemporal Capital Asset Pricing Model,” J. Bus., vol. 57 (3), 1984, pp. 337-351.

[35] H. Johnson, and D. Shanno, "Option Pricing when the Variance is Changing," J. Fin. and Quant. Anal., vol. 22 (2), 1987, pp. 143-151.

[36] P. Jorion, "On Jump Processes in the Foreign Exchange and Stock Markets," Rev. Fin. Studies, vol. 88 (4), 1989, pp. 427-445.

[37] I. Karatzas, J. P. Lehoczky, S. P. Sethi and S. E. Shreve, "Explicit Solution of a General Consumption/Investment Problem,” Math. Oper. Res., vol. 11, 1986, pp. 261-294. (Reprinted in Sethi [50, Chapter 2].)

[38] J. Kelly, “A New Interpretation of Information Rate,” Bell Sys. Tech. J., vol. 35, 1956, pp. 917926.

[39] S. G. Kou, “A Jump Diffusion Model for Option Pricing,” Mgmt. Sci., vol. 48, 2002, pp. 10861101.

[40] S. G. Kou and H. Wang, "Option Pricing Under a Double Exponential Jump Diffusion Model," Mgmt. Sci., vol. 50 (9), 2004, pp. 1178-1192.

[41] R. Lord, R. Koekkoek and D. van Dijk, "A Comparison of Biased Simulation Schemes for Stochastic Volatility Models," Tinbergen Institute Discussion Paper, 2005/2007, pp. 1-28; preprints: http://www.tinbergen.nl/discussionpapers/06046.pdf .

[42] L. C. MacLean and W. T. Ziemba, "Capital Growth: Theory and Practice," in Handbook of Asset and Liability Management, Volume 1: Theory and Methodology, 2006, pp. 429-474.

[43] R. C. Merton, "Lifetime Portfolio Selection Under Uncertainty: The Continuous-Time Case," Rev. Econ. and Stat., vol. 51, 1969, pp. 247-257. (Reprinted in Merton [48, Chapter 4].)

[44] R. C. Merton, "Optimal Consumption and Portfolio Rules in a Continuous-Time Model," J. Econ. Theory, vol. 4, 1971, pp. 141-183. (Reprinted in Merton [48, Chapter 5].)

[45] R. C. Merton, “Eratum,” J. Econ. Theory, vol. 6 (2), 1973, pp. 213-214.

[46] R. C. Merton, "Theory of Rational Option Pricing," Bell J. Econ. Mgmt. Sci., vol. 4, 1973, pp. 141-183. (Reprinted in Merton [48, Chapter 8].)

[47] R. C. Merton, “Option Pricing When Underlying Stock Returns are Discontinuous," J. Fin. Econ., vol. 3, 1976, pp. 125-144. (Reprinted in Merton [48, Chapter 9].)

[48] R. C. Merton, Continuous-Time Finance, Cambridge, MA: Basil Blackwell, 1990.

[49] R. Rishel, "Modeling and Portfolio Optimization for Stock Prices Dependent on External Events," Proc. 38th IEEE Conf. on Decision and Control, 1999, pp. 2788-2793.

[50] S. P. Sethi, Optimal Consumption and Investment with Bankruptcy, Boston: Kluwer Academic Publishers, 1997.

[51] S. P. Sethi, and M. Taksar, "A Note on Merton's "Optimal Consumption and Portfolio Rules in a Continuous-Time Model",' J. Econ. Theory, vol. 46 (2), 1988, pp. 395-401. (Reprinted in Sethi [50, Chapter 3].) 
[52] L. Scott, "Pricing Stock Options in a Jump-Diffusion Model with Stochastic Volatility and Interest Rates: Applications of Fourier Inversion Methods," Math. Fin., vol. 7 (4), 1997, pp. 413424.

[53] E. M. Stein, and J. C. Stein, "Stock Price Distributions with Stochastic Volatility: An Analytic Approach,” Rev. Fin. Studies, vol. 4 (4), 1991, pp. 727-752.

[54] J. B. Wiggins, "Option Values Under Stochastic Volatility: Theory and Empirical Estimates," J. Fin. Econ., vol. 19, 1987, pp. 351-372.

[55] G. Yan, Option Pricing for a Stochastic-Volatility Jump-Diffusion Model, Ph.D. Thesis in Mathematics, Dept. Math., Stat., and Comp. Sci., University of Illinois at Chicago, 126 pages, 22 June 2006

[56] G. Yan, and F. B. Hanson, "Option Pricing for a Stochastic-Volatility Jump-Diffusion Model with Log-Uniform Jump-Amplitudes,” Proc. 2006 American Control Conf., 2006, pp. 29892994.

[57] T. Zariphopoulou, "A Solution Approach to Valuation with Unhedgeable Risks," Finance and Stochastics, vol. 5, 2001, pp. 61-82.

[58] Z. Zhu, Option Pricing and Jump-Diffusion Models, Ph. D. Thesis in Mathematics, Dept. Math., Stat., and Comp. Sci., University of Illinois at Chicago, 17 October 2005.

[59] Z. Zhu and F. B. Hanson, "Optimal Portfolio Application with Double-Uniform Jump Model," Stochastic Processes, Optimization, and Control Theory: Applications in Financial Engineering, Queueing Networks and Manufacturing Systems/A Volume in Honor of Suresh Sethi, International Series in Operations Research and Management Science, vol. 94, H. Yan, G. Yin, Q. Zhang (Eds.), Springer Verlag, New York, NY, Invited chapter, 28 pages, June 2006. 